\title{
Interpenetrating pseudo-icosahedral clusters model for quasicrystals and consequences: asymmetry of diffraction peaks and pseudo-inflation rules
}

\author{
J.-L. Verger-Gaugry \\ LTPCM/ENSEEG/INPG (CNRS UA 29), BP 75, Domaine Universitaire, 38402 Saint \\ Martin d'Hères, France
}

\begin{abstract}
The diffraction properties of an interpenetrating pseudo-icosahedral clusters (IPIC) model of icosahedral phase are studied. The structure factor is treated in perturbation over the icosahedral symmetry, the site multiplicities, the atomic scattering factors which takes into account the chemical disorder inside clusters, cluster deformations in real space, and distortions in reciprocal space. Its pseudo-G invariant character is studied assuming a strong integrability condition. A "virtual ideal i-quasicrystal" is associated uniquely with the real i-phase, as closest object obeying a $6 \mathrm{D}$ crystallography. The clusters and the quasilattice contributions to the peak positions and intensity properties are decoupled. The inflation rules coming from the quasilattice are superposed to the pseudo-inflation rules which arise from the ideal clusters. Peaks arising from clusters are non-symmetrical. Comparison with Gapproximant crystals is made.
\end{abstract}




\section{Introduction}

Pseudo-icosahedral clusters in approximant crystals and icosahedral quasicrystals are deformed according to the constitutive atom types of the system, exhibit a chemical disorder, interpenetrate, spread over various distances, are of Mackay or Bergmann type [1-6]. In the following, we consider a formal (real but a priori arbitrary) non-periodic state of matter exhibiting IPIC (we call it an i-phase) and incident monochromatic $\mathrm{X}$-rays or high energy electrons. The present approach consists in developing the structure factor of the i-phase in a Taylor series, in perturbation over several variables. For this, we construct an "ideal i-quasicrystal" associated with the real one, in which the icosahedral symmetry is restored exactly locally, in which the chemical disorder is set to zero in each cluster, and which serves as an optimally positioned [2b] reference (infinite) set of colored (color $=$ type of atom) points to measure deviations from it in the diffraction process of the i-phase.

\section{Preliminary definitions}

We are just recalling the notations and the terminology in ref. [2a], and will use the same. $\mathbf{R}^{3}$, our physical space $E$ as embedded in $\mathbf{R}^{6}$ as an irreducible $\mathbf{R}[\mathrm{G}]$-module, is referred to an orthonormal basis and the action of $G$ in $\mathbf{R}^{3}$ is fixed once for all in this basis. A G cluster $X(n), n>0$ and integer, is a finite collection of points in $\mathbf{R}^{3}$, not included in a plane, such that: i) it is G-invariant, ii) it is a union of $n$ shells (layers), each of them constituted by the vertices of perfect regular G-invariant polyhedra. ii) is a consequence of i). The center of the cluster is included or not in $X(n)$. The polyhedra on which $\mathrm{G}$ acts transitively have names, with zero degrees of freedom: icosahedron (12 vertices), dodecahe- 
dron (20), icosidodecahedron (30), and generic names for one degree of freedom: trisicosahedron (60), hexecontahedron (60), pentakisdodecahedron (60), and two degrees of freedom: icosidodecatriacontahedron (120). Each vertex in a layer is attributed an atom, and we speak of a colored site instead of an atomic site, the color being the atom type. A colored $\mathrm{G}$ cluster $X(n)$ is here a $\mathrm{G}$ cluster where each vertex is occupied by an atom and coloration is G-invariant. This definition is a bit more restrictive than in ref. [2a], and aims at analyzing the effect of chemical disorder inside clusters. A color corresponds to an atomic radius. A deformation of a colored G cluster $X(n)=\left\{\left(x_{i}\right.\right.$, color of $x_{i}$ ) is a map $\delta$ of $X(n)$ to $\mathbf{R}^{3}$ which preserves coloration and does not change the position of the center of the $\mathrm{G}$ cluster. It is a collection of displacements $\left\{\delta\left(x_{i}\right)-x_{i}\right.$, at the site $x_{i}$ \}, such that no interpenetration of atoms occurs after deformation.

Let us now define formally an "i-phase" as recent icosahedral local order studies $[1,2,6]$ seem to suggest it. It is a state of matter in 3D Euclidean space $E$ where the following local conditions are satisfied;

i) There exists an enumerable number of sites $\mathrm{CC}=\left\{s_{1}, s_{2}, s_{3}, \ldots\right\}$ (with CC for cluster centers; and $\mathrm{CC}$ a Delaunay set) such that, for each $i$, there exists a perfect non-colored $G$ cluster $X_{i}\left(n_{i}\right)$, centered at $s_{i}$, and a finite set of atoms around $s_{i}$ which is obtained (formally) by a small deformation $\delta_{i}$ of the $\mathrm{G}$ cluster $X_{i}\left(n_{i}\right),=$ $\delta_{i}\left(X_{i}\left(n_{i}\right)\right)$; and we add the colors to the points of $\delta_{i}\left(X_{i}\left(n_{i}\right)\right)$ so that this cluster becomes identical to the local "colored" icosahedral environment at $s_{i}$.

ii) Orientational compatibility. There exists a finite number $J$ of common average clusters representing all the possible icosahedral environments in the i-phase; $\mathrm{CC}(r)$, for all $1 \leq r \leq J$, denotes the subset of $\mathrm{CC}$, assumed infinite, of the cluster centers $s_{i}$ which present an environment of $r$ th type: there exists an integer $N_{r}$, with $1 \leq N_{r} \leq n_{i}$ for all $i$ such that $s_{i}$ is in $\operatorname{CC}(r)$, such that the intersection $\bigcap_{i, s_{i}}$ in $\left.\operatorname{ccc}_{r}\right)\left[X_{i}\left(n_{i}\right)-s_{i}\right]$, where each cluster is translated to the origin, is a (non-colored) non-empty G cluster $X^{(r)}\left(N_{r}\right)$.

iii) (Covering) every atom ( $=$ colored site) in the i-phase belongs at least to one distorted colored $\delta_{i}\left(X_{i}\left(n_{i}\right)\right)$, centered at $s_{i}$, for a certain $i$.

In approximant crystals, it is possible to define, from a set constituted by a finite number of pseudo-icosahedral clusters, a perfectly icosahedral cluster optimally positioned in it $[2 \mathrm{a}, 2 \mathrm{~b}]$. Here, the number of pseudo-icosahedral clusters having their centers in $\mathrm{CO}(r)$, for each $r$, is assumed to be infinite, and we assume that we can also define an optimal perfectly icosahedral cluster in this case: for each $r$, the meaning of the reference cluster of the $r$ th family, $X^{(r)}\left(N_{r}\right)$, is that it minimizes all the deformations $\left\|\delta_{i}\right\|=$ $\operatorname{Sup}_{j}\left\|\delta_{i}\left(x_{j}\right)-x_{j}\right\|$ for $x_{j}$ running over all the sites in $X^{(r)}\left(N_{r}\right)+s_{i}$, for all $i$ such that $s_{i}$ are in $\mathrm{CC}(r)$. $\left\|\delta_{i}\right\|$ represents the "deformation amplitude" of the $i$ th pseudo-icosahedral cluster $\delta_{i}\left(X_{i}\left(n_{i}\right)\right)$.

Because interpenetration is assumed to occur everywhere (IPIC model), an atomic site belongs to several clusters at the same time. The spatial extent of the clusters is such that we have not to consider atoms between clusters, such as "glue" atoms or polyhedra for linkages, ..., because here all the atoms are "covered" by the clusters (condition iii)). In this extent, the present approach is more natural than making models with, on one side icosahedral clusters, and, on the other side, a suitable "atomic glue". This approach is followed, for instance, by Aragon et al. [7] in the decahedral recursive growth model which is an IPIC model formed by aggregation by coincidence of partial pseudoicosahedral clusters, or by Burkov [8] with more constraining rules due to the rigidity in the geometry of the clusters, for the d phase.

If $\Lambda_{j}$ denotes the site multiplicity of the $j$ th site $x_{j}$, that is, the number of distorted $\mathrm{G}$ clusters which contain this atomic site, we have that the average site multiplicity of the i-phase $\Lambda$ is given by:

$\Lambda=\lim _{R \rightarrow+\infty}\left(\sum_{x_{j} \in S_{R}} \Lambda_{j} / \#\left(j / x_{j} \in S_{R}\right)\right)$,

where $S_{R}$ denotes the sphere of radius $R$ centered at the origin. We call $\lambda_{j}=\Lambda_{j}-\Lambda$ the site multiplicity excess for the atom $j$. 


\section{Structure factor of an IPIC model of the icosa- hedral phase}

If $C_{m}, K_{m}$ denote respectively the atomic concentration of the species $m$ in the i-phase, and its atomic scattering factor, we call $K$ the average atomic scattering of the i-phase; we have $K=$ $\Sigma C_{m} K_{m}$, where the sum is over the set of the atomic species in the i-phase. $K_{m}$ can be written as the product of the atomic number $Z_{m}$ times a polynomial in $\sin (\theta) / \nu$ whose coefficients depends upon the type of the incident radiation ( $X$ or electrons) [2a,9]. Similarly, we call $K_{l k}$ the average atomic scattering factor relatively to the $l$ th icosahedral layer, denoted by $L_{l k}$, in the $k$ th cluster $X_{k}\left(n_{k}\right)$. For instance, the small icosahedron centered at $\mathrm{Ni}(e)$ sites in the $\eta-\mathrm{Ti}_{2} \mathrm{Ni}$ crystal [10], composed by $9 \mathrm{Ti}$ and $3 \mathrm{Ni}$ gives here $\mathrm{K}_{l 1}=\left(9 K_{\mathrm{Ti}}+3 K_{\mathrm{Ni}}\right) / 12$ for the first layer in the first cluster. Define now $z_{j}=K_{j}-K_{l k}$ the atomic scattering factor excess for the $j$ th atom lying in the $l$ th layer of the $k$ th cluster (with the $\sin (\theta) / \nu$ dependence).

We now consider the scattering function, with $q$ in $E^{*}$ and $0 \leqslant \gamma_{i} \leqslant 1$ :

$$
\begin{aligned}
& H\left(q, \gamma_{1}, \gamma_{2}, \gamma_{3}\right) \\
& =\sum_{r} \sum_{s_{k} \in \mathrm{CC}(r)} \sum_{l=1}^{N_{f}} \sum_{L_{l k}} \frac{\left(K_{l k}+\gamma_{l} z_{j}\right)}{\left(\Lambda+\gamma_{2} \lambda_{j}\right)} \\
& \quad \times \exp \left[2 \mathrm{i} \pi q \cdot\left(x_{j}+\gamma_{3}\left[\delta_{k}\left(x_{j}\right)-x_{j}\right]\right)\right],
\end{aligned}
$$

where the first sum (on the left) extends over the $J$ possible classes $\mathrm{CC}(r)$ of different icosahedral environments (variable $r$, with $1 \leq r \leq J$ ), the second sum extends over all the sites $s_{k}$ in the class $\mathrm{CC}(r)$, the third sum is taken over all the layers $L_{l k}$ in the $k$ th cluster (variable $l$, with $1 \leq l \leq N_{r}$ ), and the fourth sum is taken over all the atomic sites $x_{j}$ in the layer $L_{l k}$. The Bragg peak intensities of the i-phase are given by:

$$
I(q)=|H(q, 1,1,1)|^{2},
$$

for $q$ in reciprocal space $E^{*}$. Actually, we take $E=E^{*}$. Peak positions are given by the (vector) equations:

$$
\partial I /\left.\partial q\right|_{\gamma_{1}-\gamma_{2}-\gamma_{3}-1}=0 .
$$

Since icosahedral environments are treated separately in the sum (1), we will consider, for simplicity's sake, that $J$ is equal to 1 and write CC instead of $\mathrm{CO}(1)$. For $\mathrm{Pm} \overline{3} \overline{5}$ i-phases, such as i-AlMn, i-AlMnSi, i-AlCr, ..., i-AlLiCu, $J$ is probably equal to 1 , while $J>1$ for $\mathrm{Fm} \overline{3} \overline{5}$ iphases, like i-AlFeCu, i-AlPdMn, etc. with several types of icosahedral local order.

\subsection{Cluster contributions in real space}

As in the IPIC model for approximant crystals [2a], we assume that the scattering function $H$ is given by its first order series development at $(q, 0,0,0)$ :

$$
\begin{aligned}
& H\left(q, \gamma_{1}, \gamma_{2}, \gamma_{3}\right) \\
& \quad=H(q, 0,0,0)+\sum_{i=1}^{3} \partial H /\left.\partial \gamma_{i}\right|_{\gamma_{1}=\gamma_{2}=\gamma_{3}=0} \gamma_{i},
\end{aligned}
$$

neglecting second order terms. The partial derivatives of $H$ are now infinite sums over CC, which take into account each effect "linearly", namely, the scattering factor excess, the site multiplicity excess and the deformations in real space. We have:

$$
\begin{aligned}
& \partial H /\left.\partial \gamma_{1}\right|_{\gamma_{1}=\gamma_{2}=\gamma_{3}=0} \\
& \quad=\sum_{s_{k} \in \mathrm{CC}} \sum_{l=1}^{N_{f}} \sum_{L_{l k}} \frac{z_{j}}{\Lambda} \exp \left[2 \mathrm{i} \pi q \cdot x_{j}\right], \\
& \partial H /\left.\partial \gamma_{2}\right|_{\gamma_{l}=\gamma_{2}=\gamma_{3}=0} \\
& =-\frac{K}{\Lambda} \sum_{s_{k} \in \mathrm{CC}} \sum_{l=1}^{N_{f}} \frac{K_{l k}}{K} \sum_{L_{l k}} \frac{\lambda_{j}}{\Lambda} \exp \left[2 \mathrm{i} \pi q \cdot x_{j}\right],
\end{aligned}
$$

$$
\begin{aligned}
& \partial H /\left.\partial \gamma_{3}\right|_{\gamma_{1}-\gamma_{2}-\gamma_{3}-0} \\
& =\frac{2 \mathrm{i} \pi K}{\Lambda} \sum_{s_{k} \in \mathrm{CC}} \sum_{l=1}^{N_{j}} \frac{K_{l k}}{K} \sum_{L_{l k}}\left(q \cdot\left[\delta_{k}\left(x_{j}\right)-x_{j}\right]\right) \\
& \quad \times \exp \left[2 \mathrm{i} \pi q \cdot x_{j}\right] .
\end{aligned}
$$

When clusters are identical and have no chemical disorder, eq. (5) is reduced to zero: $z_{\mathrm{uw}(l)}$, the average scattering factor excess over all the atomic sites on the lth layer of each cluster, is equal to 
zero. When clusters are not identical and present a chemical disorder, eq. (5) denotes an error term with respect to the icosahedral group action in the cluster. The ideal G-invariant coloration of the cluster $X^{(r)}\left(N_{r}\right)$, representing the class CC $=$ $\mathrm{CO}(r)$, with $r=1$, is therefore given, on the $l$ th layer, by the scattering factor $K_{\mathrm{av}(t)}$, which is not necessarily a "pure color" (corresponding to a unique atom type), where $K_{\operatorname{av}(t)}$ is the average value of $K_{l k}$ over all the atomic sites on the $l$ th layer of every cluster and over all the clusters centered in CC.

Similarly, if $\lambda_{\operatorname{av}(l)}$ is the average site multiplicity excess over al the atomic sites on the $l$ th layer of every cluster and over all the clusters centered in CC, the sum (6) is decoupled into a cluster term and a cluster center distribution term assumed to be convergent, and is equal to, at the first order

$$
\begin{aligned}
- & \frac{K}{\Lambda}\left(\sum_{i=1}^{N_{r}} \frac{K_{\mathrm{av}(l)}}{K} \frac{\lambda_{\mathrm{av}(l)}}{\Lambda} \sum_{L_{i k}} \exp \left[2 \mathrm{i} \pi q \cdot\left(x_{j}-s_{k}\right)\right]\right) \\
& \times\left(\sum_{s_{k} \in \mathrm{CC}} \exp \left[2 \mathrm{i} \pi q \cdot s_{k}\right]\right) .
\end{aligned}
$$

The first factor (apart from the constant term) is real and $G$-invariant since perfectly icosahedral clusters are centrosymmetric, and the second factor is analyzed below. When interpenetration occurs uniformly with a constant site multiplicity for all the atoms, eq. (6) is exactly equal to eq. (8).

In the same way, we define $\delta_{\mathrm{av}(t)}$ to be the average deformation function restricted to the $l$ th layer, as an average over all the atomic sites on the $l$ th layer of every cluster and over all the clusters centered in CC. eq. (7) reduces, at the first order, to:

$$
\begin{aligned}
& \frac{2 \mathrm{i} \pi K}{A}\left(\sum_{l=1}^{N_{r}} \frac{K_{\operatorname{av}(l)}}{K}\left(q \cdot\left[\delta_{\operatorname{av}(l)}\left(x_{j}\right)-x_{j}\right]\right)\right. \\
& \left.\quad \times \sum_{L_{l k}} \exp \left[2 \mathrm{i} \pi q \cdot\left(x_{j}-s_{k}\right)\right]\right) \\
& \quad \times\left(\sum_{s_{k} \in \mathrm{CC}} \exp \left[2 \mathrm{i} \pi q \cdot s_{k}\right]\right),
\end{aligned}
$$

with the $j$ index in $\delta_{\text {av }(l)}\left(x_{j}\right)-x_{j}$, and $x_{j}$ any site in a layer $L_{l k}$. The first factor is here also real (apart from the front coefficient) and G-invariant. When all the cluster deformations are normal to the $q$ direction, eq. (7), as eq. (9), gives a zero contribution.

So far, from eq. (4), the difference between $H(q, 1,1,1)$ and $H(q, 0,0,0)$ is only given, at the first order, by the sum of the contributions (8) and (9).

\subsection{Cluster centers and quasilattice contributions in real space}

At which or around which $q$ do we have intense peaks? We will sketch the transformation of the sum $\sum_{s_{k} \in C C} \exp \left[2 \mathrm{i} \pi q \cdot s_{k}\right]$, considering that $E$ is embedded in $\mathbf{R}^{6}$ as the image of the orthogonal projector $P_{\|}$, along the perpendicular (internal) space $E_{\perp}$, with $\mathbf{R}^{6}=E \oplus E_{\perp}$, in order to answer to this question. We know that we have only three classes of $\mathrm{G}$ invariant lattices in $\mathbf{R}^{6}$, up to quasihomothety: the simple cubic one, the fcc and the bec [11]. The relationships between $6 \mathrm{D}$ $\mathrm{G}$-invariant lattices and the set of the cluster centers $\mathrm{CC}$ are a priori not obvious and two main behaviours for $\mathrm{CC}$ can occur: 1) $\mathrm{CC}$ is exactly included in the projection by $P_{\|}$of a $6 \mathrm{D} \mathrm{G}$-invariant lattice, 2) CC is not included in the projection by $P_{\|}$of any 6D G-invariant lattice. In the first case, we take the smallest $6 \mathrm{D}$ G-lattice $L$ whose projection by $P_{\|}$contains $\mathrm{CC}$ and each element $s_{k}$ of CC has a unique lifting, denoted by $c_{k}$, in $L$, with $c_{k \|}=s_{k}$. In the second case, there is an ambiguity; we fix $1 \gg \epsilon>0$ an arbitrary real number and take any 6D G-invariant lattice $L$ : its projection by $P_{\|}$is dense and each center in $\mathrm{CC}$ can be approached by an element of $P_{\|}(L)$, within a distance less than $\epsilon$. We choose an element $c_{k}$ in $L$ such that $\left\|c_{k+}\right\|$ is of minimal norm in $E_{\perp}$, and that $\left\|c_{k \|}-s_{k}\right\|<\epsilon$. In both cases, we say that the i-phase is bandable when there exists a constant $B<\infty$ such that, for all $k$, $\left\|c_{k \perp}\right\|<B$, when $\epsilon$ tends to 0 . If not, there is a "geometric divergence" from $E$ for the set $\left\{c_{k}\right\}$. Such a divergent behavior was recently studied in (quasi $^{2}$-crystals by Lançon and Billard [12]. We now use the properties of modulo 1 equireparti- 
tion to change the summation into an integral, with $g$ in the $6 \mathrm{D}$ lattice $L^{*}$ dual to $L$ in $\mathbf{R}^{6}$ and $g_{\|}=q$ :

$$
\begin{aligned}
& \lim _{R \rightarrow>+\infty}\left(\frac{1}{\#\left\{k / s_{k} \in \mathrm{CC} \cap S_{R}\right\}} \sum_{s_{k} \in \mathrm{CC} \cap s_{R}}\right. \\
& \left.\quad \times \exp \left[2 \mathrm{i} \pi q \cdot s_{k}\right]\right)=\int \exp \left(-2 \mathrm{i} \pi g_{\perp} \cdot y\right) \mathrm{d} y,
\end{aligned}
$$

where the integral is taken over the volume $V_{\mathrm{CC}}$ defined by the adherence of the enumerable set $P_{\perp}\left(\left\{c_{k}\right\}\right)$ in $E_{\perp}$. Since we are interested in IPIC models for which the intensities in reciprocal space are convergent and follow the icosahedral symmetry, this integral should converge; $V_{\mathrm{CC}}$ should also be G-invariant for the action of $G$ in $E_{\perp}$ as it will appear in the decomposition of $H(q, 0,0,0)$. For bandable i-phase structures, the integrability condition is always satisfied since $V_{\mathrm{CC}}$ is bounded, and we obtain the Fourier transform of the window $V_{\mathrm{CC}}$. We assume that this integrability condition and the G-invariance of $V_{\mathrm{CC}}$ are satisfied in the following.

\subsection{Inflation and pseudo-inflation rules}

The exponential sum $H(q, 0,0,0)$ can be written, at the first order:

$$
\begin{aligned}
& \frac{K}{\Lambda}\left(\sum_{l=1}^{N_{\prime}} \frac{K_{\mathrm{av}(l)}}{K} \sum_{L_{i k}} \exp \left[2 \mathrm{i} \pi q \cdot\left(x_{j}-s_{k}\right)\right]\right) \\
& \quad \times\left(\sum_{s_{k} \in C \mathrm{C}} \exp \left[2 \mathrm{i} \pi q \cdot s_{k}\right]\right),
\end{aligned}
$$

which is the product, apart from the constant term, of a real G-invariant factor attached to the average cluster of the class $\mathrm{CC}$, by a quasilattice contribution assumed convergent. Grouping now the eqs. (8), (9), (10) and (11), $H(q, 1,1,1)$ is, at the first order, given asymptotically by:

$$
\begin{aligned}
\lim _{R \rightarrow+\infty}\left(\frac{1}{\#\left\{k / s_{k} \in \mathrm{CC} \cap S_{R}\right\}} H(q, 1,1,1)\right) \\
=K S_{c l}(q) T / \Lambda .
\end{aligned}
$$

where

$$
\begin{aligned}
S_{c l}(q) & \\
= & \left(\sum _ { l = 1 } ^ { N r } \frac { K _ { \operatorname { a v } ( l ) } } { K } \left(1+2 \mathrm{i} \pi\left(q \cdot\left[\delta_{\operatorname{av}(l)}\left(x_{j}\right)-x_{j}\right]\right)\right.\right. \\
- & \left.\left.\frac{\lambda_{\mathrm{av}(l)}}{A}\right) \sum_{L_{l k}} \exp \left[2 \mathrm{i} \pi q \cdot\left(x_{j}-s_{k}\right)\right]\right)
\end{aligned}
$$

is G-invariant, where $T$ is the G-invariant transparency function given by eq. (10) associated with the cluster distribution in the hyperspace $\mathbf{R}^{6}, q$ in the projection $P_{\|}\left(L^{*}\right)$. The clusters have their own diffracting properties and exhibit pseudo-inflation rules, and they appear by the term $S_{c l}(q)$; both contributions - quasilattice and clusters are superposed, the first ones being $\delta$-peaks and the other ones non-symmetrical peaks from ideal finite clusters [13], able to give extinctions. Finally, from eq. (2), restituting the $J$ possible classes of icosahedral environment:

Proposition 1: For any IPIC model of the iphase, the intensity $I(q)$ is given by, at the first order, by the G-invariant expression:

$I(q)=\left|K / \Lambda\left(\sum_{r=1}^{J} S_{c l}^{(r)}(q) T_{(r)}\right)\right|^{2}$,

for $q$ in $P_{\mathrm{f}}\left(L^{*}\right)$ and zero elsewhere.

The $3 \mathrm{D}$ virtual ideal $\mathrm{i}$-phase is given by the set of cluster centers $P_{-}\left(\left\{c c_{k}^{(r)}\right\}\right)$, close to $\mathrm{CC}(r)$, and the average ideal cluster $X^{(r)}\left(N_{r}\right)$ with the coloration $K_{\text {av }(t)}^{(r)}$. Its 6D associated object is immediately obtained if we assume that the layer radii in $X^{(r)}\left(N_{r}\right)$ are rational. The continuity hypothesis for the atomic surfaces proposed by Katz [14] allows, by periodical extension in $L$ (which can be taken common for all classes), the constructions of the corresponding ideal $6 \mathrm{D} \mathrm{i}$-crystal and the corresponding atomic surfaces.

\section{Conclusion}

We have shown how the structure factor can be calculated from the interpenetrating pseudoicosahedron cluster model for quasicrystals, at 
least at the first order, and indicate some of the relevant variables in its series development.

\section{References}

[1] N. Tamura and J.-L. Verger-Gaugry, J. Non-Cryst. Solids $153 \& 154$ (1993) 546.

[2] a) J.-L. Verger-Gaugry, J, de Phys. I 1 (1991) 1303; b) J.-L. Verger-Gaugry, N. Tamura and J.-N. Barbier, to be submitted to J. de Phys. I; c) J.-L. Verger-Gaugry, to be submitted to J, de Phys. I.

[3] D.P. Divicenzo and P.J. Steinhardt, Quasicrystals - The State of the Art, Directions in Condensed Matter Physics 11 (World Scientific, 1991).

[4] J.L. Robertson, in: Methods of Structural Analysis of Modulated Structures and Qausicrystals, eds. J.M.
Perez-Mato, F.J. Zuniga and G. Madariaga (World Scientific, 1991) p. 521.

[5] K. Sekimoto, in: Quasicrystals, eds. T. Fujiwara and T. Ogawa, Springer Series in Solid-State Sciences 93 (Springer, 1990) p. 120.

[6] C. Henley, ref. [5] p. 38.

[7] J.L. Aragon, D. Romeu and A. Gomez, ref. [4] p. 648.

[8] S.E. Burkov, ref. [4] p. 531.

[9] K. Lonsdale (ed.), Int. Tables for X-ray Crystallography (Reidel, Dordrecht, 1983).

[10] N. Tamura, A. François, A. Loiseau and J.-L. VergerGaugry, Phil. Mag. B, submitted.

[11] L.S. Levitow and J. Rhyner, J. de Phys. 49 (1988) 1835.

[12] F. Lançon and L. Billard, MOSPOQ'91 at Balatonszeplak, Hungary, in press.

[13] N. Tamura, J.-N. Barbier and J.-L. Verger-Gaugry, in preparation.

[14] A. Katz, in: Number Theory and Physics II, eds. by J.M. Luck, P. Moussa and M. Waldschmidt, (Springer, 1992). 